Wandel in der Negation. In all diesen Fällen wird erst die Situation im Neuhochdeutschen dargelegt, danach wird auf das Althochdeutsche zurückgegangen und dann der Wandel vom Althochdeutschen zum Neuhochdeutschen analysiert. So ist zum Beispiel der Genitiv sowohl in adverbialer (Komplement-)Funktion als auch in adnominaler (Attributiv-)Funktion vom Althochdeutschen zum Neuhochdeutschen trotz präskriptiven Drucks der Grammatikschreibung der letzten Jahrhunderte stark zurückgegangen. Die vermutlichen Ursachen dieses Rückgangs sind jedoch sehr komplex und offen für verschiedene Hypothesen und Interpretationen, wobei auch die methodologischen Probleme, die im ersten Teil vorgestellt wurden, eine Rolle spielen. Auf diese Art und Weise lesen sich viele Kapitel im mittleren Teil wie kleine Krimis. Es ist Spurensuche und Ursachenforschung für Tatsachen, mit denen man sich als Resultat konfrontiert sieht.

Die fünf Erklärungsmodelle im letzten Teil sind präskriptive Normierungen, Sprachkontakt, funktionale Erklärungen, formale Ansätze und optimalitätstheoretische Ansätze. Die beiden letzteren, formallinguistischen Kapitel sind vom KoAutor Oliver Schallert verfasst. Die drei ersten Kapitel vom Hauptautor nehmen sich eher konservativ aus. In der allgemeinen Sprachwissenschaft gibt es auch verschiedene theoretische Ansätze zum Sprachwandel, aber diese drei Kapitel beruhen hauptsächlich auf der stolzen Tradition der Germanistik. In diesem Sinne sind sie jedoch sehr fundiert geschrieben.

Insgesamt war ich überrascht, wie spannend und informativ dieses Buch ausgefallen ist - Sprachgeschichte kann ja auch leicht sehr trocken und langweilig werden. Insgesamt ist es eine große Leistung der Autoren. Ein Caveat jedoch: Unter Historische( $r$ ) Syntax des Deutschen könnte man auch eine historische Beschreibung der Syntax des Deutschen verstehen. Darum geht es in diesem Buch jedoch nicht. Vielmehr handelt es sich um eine Einführung in die, oder Anleitung zu der, Erforschung der historischen Syntax des Deutschen.

\title{
Fobbe, Eilika:
}

Forensische Linguistik. Eine Einführung. Tübingen: Narr, 2011 (narr studienbücher). - ISBN 978-3-8233-6654-6. 250 Seiten, € 19,90

\section{(Barbara Vogt, Trieste / Italien)}

Das Buch führt in eine verhältnismäßig junge Disziplin der angewandten Sprachwissenschaft, die forensische Linguistik, ein, die ganz allgemein »alles Sprachliche im Bereich des Rechts « (16) untersucht. Es liegen verschiedene Versuche vor, die Forschungsfelder der forensischen Linguistik zu erfassen und zu systematisieren. Beispielsweise bietet sich eine Differenzierung in Rechtslinguistik, also die Sprache als Instrument der Justiz, und in forensische Linguistik an, wobei letztere von Fobbe als Hilfswissenschaft, die sich auf »sprachliche Produkte, die für 
Ermittlungen oder für zivil- oder strafrechtliche Verfahren relevant sind und ggf. einen Beweiswert besitzen « (16), bestimmt wird. In dem vorliegenden Buch konzentriert sich die Autorin auf den letztgenannten Bereich und widmet sich speziell der linguistischen Analyse geschriebener Texte.

In Teil I des Buches werden der Gegenstandsbereich und die Anwendungsfelder der forensischen Linguistik vorgestellt. Es wird Grundlegendes zum Dreieck Text, Autor und Leser gesagt und es wird aufgezeigt, in welcher Hinsicht hier rechtlich relevante Sachverhalte entstehen können. So werden beispielsweise die verschiedenen Formen der Autorschaft und deren Merkmale dargelegt, und es wird erläutert, wie diese mit bestimmten Tatschreiben korrelieren. Für Erpresser- oder Drohbriefe ist beispielsweise charakteristisch, dass der Autor anonym bleiben will oder dass er vorgibt, ein anderer, ein »postulierter « Autor zu sein. In Plagiatsfällen dagegen wird der Text vom empirischen Autor keiner anderen Person zugeschrieben, der »Täter « behauptet vielmehr, den Text eines anderen selbst geschrieben zu haben.

Im zweiten Teil des Buches werden Einzelaspekte der Analyse erklärt, wobei es um die Bereiche Textsorte, Stil, Fehler und Inhalt geht. Hier gibt Fobbe Aufschluss darüber, wie die verschiedenen Textsorten aufgrund charakteristischer Merkmale (z. B. textsortenspezifischer Formulierungsmuster) klassifiziert und in eine Typologie inkriminierter Texte eingeordnet werden können. Dabei orientiert sich Fobbe am kommunikativ-pragmatischen Ansatz von Brinker (2010) und stellt exemplarisch die Textsorte Erpresser- und Abschiedsbrief vor.

Die Stilanalyse bezeichnet Fobbe - neben der Fehleranalyse - als »Kern einer forensischen Textanalyse « (107). Wie die Autorin erläutert, geht die forensische Linguistik von einem weiten Stilkonzept aus, das Stil allgemein als »Wahl« begreift, wobei die Wahl-Möglichkeiten auf allen sprachlichen Ebenen angesiedelt sind, allerdings von der Sprachstruktur, der Individualität des Sprechers/Schreibers, der Textsorte etc. limitiert werden. Im Anschluss an eine Einführung in das Stilkonzept der forensischen Linguistik stellt Fobbe einzelne Aspekte der Stilmerkmale dar, z. B. die Möglichkeiten ihrer Validierung und ihrer Bündelung zu Merkmalskomplexen.

In Kapitel 5 wird die Fehleranalyse behandelt, die in der forensischen Linguistik ebenfalls eine wichtige Rolle spielt. Dabei werden Fehler zunächst allgemein als Abweichung von einer Norm bestimmt. Es wird zwischen Fehlern von Muttersprachlern und Nicht-Muttersprachlern, also lernersprachlichen Fehlern, unterschieden. Schließlich können lernersprachliche Fehler auch von einem Muttersprachler vorgetäuscht werden, um über die wahre Identität hinwegzutäuschen. Bei der Identifizierung und Beurteilung von lernersprachlichen Fehlern bzw. fingierten lernersprachlichen Fehlern kann auf Erkenntnisse des Spracherwerbs zurückgegriffen werden. So geht die Fremdsprachen-Forschung heute oft von dem Konzept der Interlanguage aus, die die dynamische, sich entwickelnde Sprachform des Lerners darstellt. Es wird angenommen, dass ein Lerner beim Spracherwerb 
bestimmte, fixe Sequenzen durchläuft, die denen im Erstsprachen-Erwerb ähnlich sind. Weist ein Text also bestimmte Fehler auf den ersten Erwerbsstufen auf (z. B. falsche Verbkonjugation im Präsens) und verwendet aber an anderer Stelle korrekt einen Nebensatz mit komplexem Prädikat, so kann dies ein Hinweis darauf sein, dass der Schreiber nur vorgibt, kein Muttersprachler zu sein.

Der letzte Aspekt forensischer Textanalyse, den Fobbe detailliert vorstellt, betrifft den Inhalt, d. h. die Glaubwürdigkeit der zu analysierenden Texte, was insbesondere bei Zeugenaussagen und Geständnissen von Bedeutung ist. Es werden verschiedene Kriterien für das Vorliegen einer »Lüge« sowie moralisch-philosophische, psychologische und pragmatische Beschreibungsansätze für falsche Aussagen eingeführt. Mit dem Grad der Glaubwürdigkeit einer Aussage befasst sich die Aussagenpsychologie. Hier stellt Fobbe die merkmalsorientierte Inhaltsanalyse vor (criteria-based content-analysis, $C B C A$ ), die mit Hilfe eines Kriterienkatalogs, der sowohl auf die Textstruktur als auch den Inhalt der Aussage abzielt, die Glaubwürdigkeit von Aussagen bestätigen soll.

Im letzten Teil widmet sich Fobbe schließlich der Frage, in welcher Form ein Linguist die Ergebnisse seiner Analyse darlegen kann. So kann ein Sprachwissenschaftler als Gutachter vor Gericht oder aber auch für eine der Parteien tätig werden. Diesbezüglich werden in diesem letzten Teil schließlich auch die prozessrechtlichen Rahmenbedingungen für einen gutachterlichen Auftrag dargelegt.

Der Anhang besteht aus drei Kriminalfällen. Die Täter wurden gefasst und gestanden anschließend, die analysierten Briefe geschrieben zu haben, so dass die aus der linguistischen Analyse resultierenden Annahmen mit dem realen Autor und dem tatsächlichen Tatverlauf konfrontiert werden können.

Das Buch führt in einen spannenden Anwendungsbereich der Sprachwissenschaft ein und ist auch für juristische Laien gut verständlich. Zu jedem Kapitel werden Aufgaben formuliert, die sich auf originale Textbeispiele stützen, so dass der Leser Einblicke in die verschiedenen Formen inkriminierter Texte und die Möglichkeiten ihrer Analyse gewinnen kann. Mit vielen Analysebeispielen wird schließlich die Arbeit eines forensischen Linguisten anschaulich gemacht. Kritische Aspekte forensischer Textanalyse werden nicht ausgeblendet und ihre Grenzen aufgezeigt. So führt die Autorin beispielsweise aus, dass insbesondere die Möglichkeiten einer quantitativen Stilanalyse anfangs überschätzt wurden. Sprachliche Produkte sind eben nicht mit »Fingerabdrücken« zu vergleichen, denn Sprache ist veränderlich und situationsabhängig. Auf der anderen Seite zitiert die Autorin aber auch Fälle, bei denen ein linguistisches Urteil dabei geholfen hat, Fehlurteile zu revidieren.

\section{Literatur}

Brinker, Klaus: Linguistische Textanalyse. Eine Einführung in Grundbegriffe und Methoden. Berlin: Erich Schmidt, 2010. 